The Astrophysical Journal, 283:L41-L44, 1984 August 15

(1) 1984. The American Astronomical Society. All rights reserved. Printed in U.S.A.

\title{
ON THE INTERPRETATION OF THE BROAD-BAND MILLIMETER-WAVE FLUX FROM ORION
}

\author{
E. C. Sutton, ${ }^{1}{ }^{\text {Geoffrey A. Blake, }}{ }^{2}$ C. R. Masson, ${ }^{1}$ and T. G. Phillips ${ }^{1}$ \\ Received 1984 April 3; accepted 1984 May 31
}

\begin{abstract}
Spectral observations of the core of Orion A at wavelengths around $1.3 \mathrm{~mm}$ show a high density of strong, broad emission lines. The combined flux in lines with peak antenna temperatures stronger than $0.2 \mathrm{~K}$ accounts for approximately $40 \%$ of the broad-band millimeter-wave flux from the region. Thus the broad-band flux from Orion $\mathrm{A}$ is in large part due to sources other than dust emission.
\end{abstract}

Subject headings: interstellar: matter - nebulae: Orion Nebula

\section{INTRODUCTION}

The broad-band submillimeter flux from Orion $\mathrm{A}$ has been studied by Keene, Hildebrand, and Whitcomb (1982) and by Elias et al. (1978). Taken together, their data imply a flux density of about $128 \mathrm{Jy}$ at $1 \mathrm{~mm}$ in a $0^{\prime} 6$ beam with a spectral index of about 3. This has generally been interpreted as optically thin emission from dust grains, the steep spectral index being in part due to the steep dust opacity law.

It has been known also that Orion has emission from a large number of strong molecular lines. Recently, Sutton et al. (1984) have systematically surveyed the line emission between 215 and $247 \mathrm{GHz}$. The data show several hundred strong, broad emission lines which in several places blend together to obscure any underlying continuum. The combined emission from these lines is equivalent to that of a continuum with antenna temperature of $0.25 \mathrm{~K}$ for a 0.6 beam, which is generally comparable to the total broad-band flux from the region. Thus it is important to examine carefully the calibrations of these data in order to determine whether the bulk of the submillimeter flux should be attributed to line emission instead of dust emission.

\section{RESULTS AND CALIBRATION}

\section{a) Emission-Line Flux Density}

The data of Sutton et al. (1984) show over 500 resolvable lines in the frequency interval $215-247 \mathrm{GHz}$ ranging in temperature from about $100 \mathrm{~K}$ (the $J=2-1$ line of ${ }^{12} \mathrm{CO}$ ) down to the noise level of about $0.2 \mathrm{~K}$. A compressed view of the spectrum is presented in Figure 1. Line widths present vary from $\sim 4 \mathrm{~km} \mathrm{~s}^{-1}(3 \mathrm{MHz})$ for the "spike" components of the gas out to $\sim 100 \mathrm{~km} \mathrm{~s}^{-1}$ (77 MHz) for the broad "plateau" emission. Approximately half of the lines have an intermediate $\sim 12 \mathrm{~km} \mathrm{~s}^{-1}(9 \mathrm{MHz})$ line width and are attributed to material in the Orion "hot core." Averaged over the frequency range searched, these lines contribute approximately $0.25 \mathrm{~K}$ to the broad-band emission. The $J=2-1$ line of ${ }^{12} \mathrm{CO}$ alone contributes $\sim 0.05 \mathrm{~K}$ of this emission.

\footnotetext{
${ }^{1}$ Department of Physics, California Institute of Technology.

${ }^{2}$ Department of Chemistry, California Institute of Technology.
}

The spectral measurements were calibrated using a standard chopper wheel technique, with beam efficiencies determined by observing the Moon and planets. Sutton et al. (1984) reduced their data to a scale of corrected antenna temperature using an efficiency of 0.85 , appropriate for emission from spatially extended sources (sources several times larger than the 0.5 beam). Since the "hot core" and "plateau" lines arise from more compact regions, a lower efficiency factor should be used to derive proper main beam brightness temperatures for these components. In general, because of the different sized emitting regions it will not be possible to apply a single flux density or temperature scale which is appropriate for all the lines.

For the present discussion, line fluxes have been calibrated directly from measurements of Saturn. Saturn is small compared to the main beam, a fact which is also known to be true for the plateau component in Orion (Plambeck et al. 1982; Friberg 1984; Masson et al. 1984). Plateau emission dominates the line fluxes seen here. As a typical example of the calibration, in 1983 May Saturn subtended a diameter of 0.30. Assuming a physical temperature of $160 \mathrm{~K}$, this implies a flux density at $1.3 \mathrm{~mm}$ of $1500 \mathrm{Jy}$. The measured antenna temperature of Saturn under these conditions was $13.3 \mathrm{~K}$ (no correction for efficiency). Measurements of Venus and Jupiter (the latter is partially resolved by the main beam) provide consistent calibration within $\pm 20 \%$. With this calibration the average flux due to lines stronger than $0.2 \mathrm{~K}$ is $25 \mathrm{Jy}$ at a flux-weighted mean wavelength of $1.3 \mathrm{~mm}$. The limited 260 $\mathrm{GHz}$ data of Blake et al. (1984) clearly show much stronger line emission, as would be generally expected for lines arising in regions of high excitation. With a similar calibration, the average line flux density at $1.15 \mathrm{~mm}$ is $51 \mathrm{Jy}$. These results are plotted in Figure 2.

\section{b) Broad-Band Flux Density}

The total broad-band flux (lines plus continuum) from Orion can be determined from the same set of measurements. For comparison with the line fluxes, this is the best procedure since the observations were made with the same beam size, wavelength, and pointing errors as the spectral line data. 


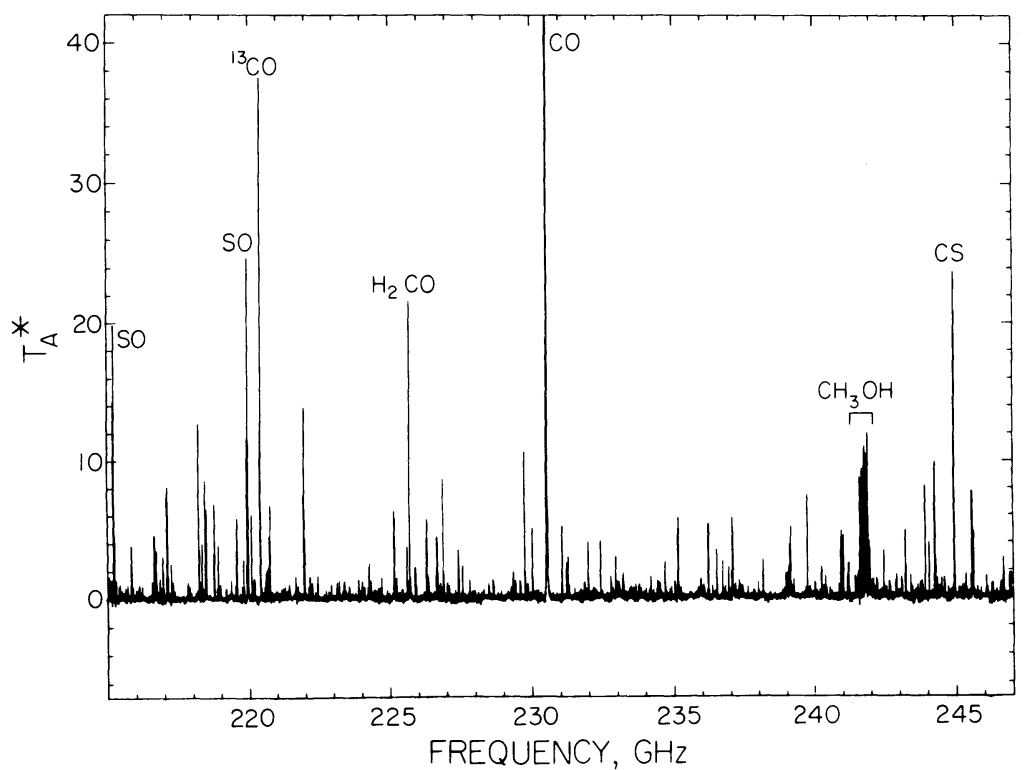

FIG. 1. - Spectrum of Orion A from 215 to $247 \mathrm{GHz}$. Data were obtained with a typical receiver noise temperature of $500 \mathrm{~K}$ (SSB) and backend coverage of $527 \mathrm{MHz}$. The original spectral resolution was $1.03 \mathrm{MHz}\left(1.3 \mathrm{~km} \mathrm{~s}^{-1}\right)$. Spectral resolution in this plot is degraded by about a factor of 10 .

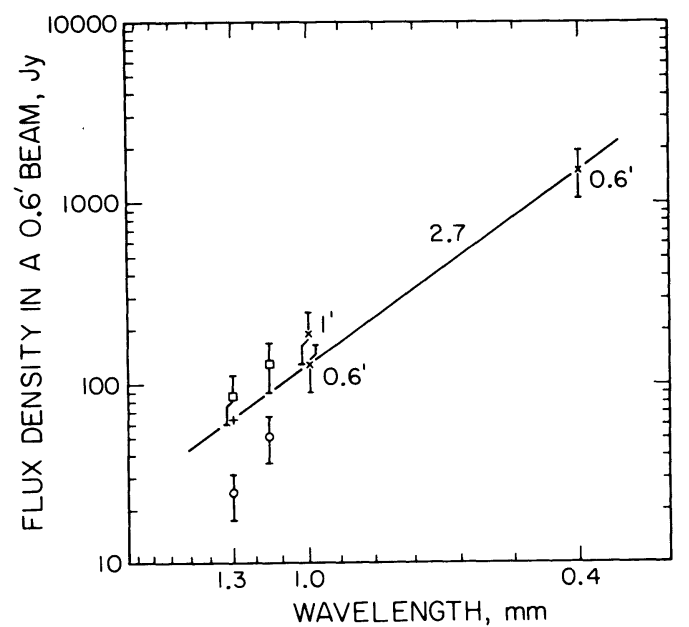

Fig. 2.-Broad-band and spectral line flux densities in a 0.6 beam centered on BN. Open circles show the line fluxes measured by Sutton et al. (1984) and Blake et al. (1984). Open boxes indicate the total broad-band fluxes measured simultaneously. Crosses represent the broadband fluxes of Keene, Hildebrand, and Whitcomb (1982) and Elias et al. (1978), the latter both for a 1' beam and corrected for a 0'6 beam. Plus sign represents the extrapolation of these short wavelength measurements to $1.3 \mathrm{~mm}$.

However, some additional uncertainties were introduced since the spectral line system was not well optimized for continuum observations. The measured broad-band flux was $86 \mathrm{Jy}$ at 1.3 $\mathrm{mm}$ with an uncertainty of about $\pm 15 \%$ on top of an overall calibration error of $\pm 20 \%$.

This value for the broad-band flux can be compared with the various values in the published literature. The $400 \mu \mathrm{m}$ emission from the core of Orion A has been measured and mapped with 0'6 resolution by Keene, Hildebrand, and Whitcomb (1982). They show that the submillimeter emission consists of an extended component plus two major compact sources. The flux density at $\mathrm{BN}$ is $1500 \mathrm{Jy}$ within a $0^{\prime} 6$ beam, with an overall calibration uncertainty of $\pm 30 \%$. Previously, the $1 \mathrm{~mm}$ emission from Orion was measured by Westbrook et al. (1976) and recalibrated by Elias et al. (1978). They derived a flux density of $188 \mathrm{Jy}$ in a $1^{\prime}$ beam. Assuming that the $1 \mathrm{~mm}$ emission has the same spatial distribution as the 400 $\mu \mathrm{m}$ emission, it is possible to estimate the $1 \mathrm{~mm}$ flux density in a 0 ' 6 beam by using the mapping data of Keene, Hildebrand, and Whitcomb (1982). The result is an estimated flux density of $128 \mathrm{Jy}$ for the smaller beam. Combined with the $400 \mu \mathrm{m}$ result, this implies a spectral index of 2.7 between $0.4 \mathrm{~mm}$ and $1 \mathrm{~mm}$. This is similar to but smaller than the spectral index estimated by Elias et al. (1978). Extrapolating with this spectral index to $1.3 \mathrm{~mm}$, the wavelength of the spectral line observations, gives a flux density of $63 \mathrm{Jy}$ in a 0.6 beam. The various broad-band fluxes are plotted in Figure 2. Comparison with the line flux indicates that $30 \%-40 \%$ of the total flux may be accounted for as line emission. This, however, is a lower limit since the line flux is based only on those lines stronger than $0.2 \mathrm{~K}$.

\section{DISCUSSION}

\section{a) Extrapolation to Weaker Lines}

The calculations above have considered only those lines strong enough to be individually detected, i.e., those with peak antenna temperatures stronger than about $0.2 \mathrm{~K}$. There will be a large number of weaker lines present which cannot be seen because of the noise level in the spectrum and because of the overlapping of stronger lines. Even at the $0.2 \mathrm{~K}$ level there are large regions of the spectrum in which the baseline cannot be clearly seen due to the overlapping wings of strong lines.

Some weaker lines can be predicted with some precision because they involve transitions with weaker matrix elements or are between more highly excited states of well-known species. But the general problem of predicting the number of 
weaker lines cannot be solved precisely, since there will be contributions from new molecular species with completely unknown abundances. A naive approach is to examine the distribution of line intensities and to extrapolate to weaker values. This distribution has been calculated for the 215-247 $\mathrm{GHz}$ data and is presented in Figure 3. The number of lines falls off steadily with increasing antenna temperature with a more rapid falloff above about $10 \mathrm{~K}$ as expected due to saturation effects. At the low-temperature end there seems to be a serious undercounting of lines in the $0.25-0.5 \mathrm{~K}$ interval. The slope at the low-temperature end is around -0.8 , indicating a converging contribution from the weaker lines. The indicated extrapolation predicts that the line flux has been underestimated by about $30 \%$ by not including the weaker lines. This raises the line contribution to $\sim 45 \%$ of the total flux.

This treatment is somewhat uncertain since it does not take into account the variety of line widths present. The individual statistics for plateau, hot core, and spike components are unfortunately insufficient to determine their distributions separately. However, it is likely that the missing lines in the low-temperature bins are mostly broad plateau lines, since weak broad lines are the easiest to miss in a crowded spectrum with a slowly varying baseline. If it is assumed that the missing lines are from the plateau source, then the line flux should be increased by a factor of about 1.7 , and it would then account for $60 \%$ of the total flux.

\section{b) Spectral Index}

The spectral index determined from the broad-band flux data is about 2.7. A similar index also applies to the spectral line data, although the value is somewhat less certain due to the limited wavelength range and the possible biases introduced by a few strong lines and bands (e.g., the $J=2-1$ line of $\mathrm{CO}$ and the $J=5-4$ band of $\mathrm{CH}_{3} \mathrm{OH}$ ).

The observed spectral index can be readily explained either by dust emission or line emission. For example, the slope is nearly that expected for optically thin emission from warm dust grains $\left(T_{d} \gtrsim 20 \mathrm{~K}\right)$ with a dust emissivity law of $\lambda^{-1}$. The line emission estimate is more difficult due to the complex ensemble of molecules present. For each type of molecule the variation in line flux with frequency will depend on the symmetry of the molecule and its column density (and hence optical depth). A linear molecule will have a series of evenly spaced lines, corresponding to the different values of $J$, the total angular momentum, whose optical depths vary as $\nu^{2}$. For a fixed velocity dispersion the line widths also vary as $\nu$. Thus the spectral flux variation for such a molecule could range from $\nu^{3}$ (optically thick) to $\nu^{5}$ (optically thin) for the case of thermal excitation to a temperature $T_{\text {ex }} \gtrsim 20 \mathrm{~K}$. Lack of adequate excitation will tend to flatten the spectrum at sufficiently high frequencies. Symmetric and asymmetric top molecules will show a more rapid flux variation, since for large values of $J$ the line strength of an entire $J \rightarrow J-1$ band will vary approximately as $J^{2}$ instead of as $J$ for a linear molecule. Thus the spectral index data can be readily explained by a mixture of various molecular species with various optical depths. Since the line flux is dominated by asymmetric top molecules such as $\mathrm{SO}_{2}$, the relatively low index of 2.7 suggests most of the emission is in optically thick lines.

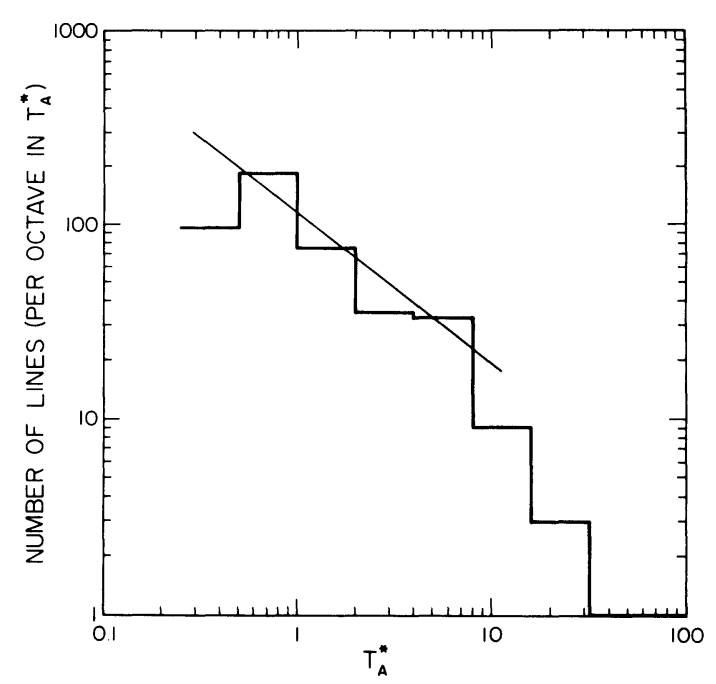

FIG. 3.-Distribution of peak corrected antenna temperatures for lines in the $215-247 \mathrm{GHz}$ band.

\section{c) Source Size}

An estimate of the size of the Orion plateau source may be obtained for the $\mathrm{SO}_{2}$ lines from the conclusion that the strongest of these are optically thick. The strongest $\mathrm{SO}_{2}$ lines have peak antenna temperatures of $\sim 20 \mathrm{~K}$. Since the excitation temperature for $\mathrm{SO}_{2}$ is thought to be about $90 \mathrm{~K}$ (Schloerb et al. 1983) this implies a source size of approximately 14" diameter. This is in good agreement with previous measurements of the plateau size (Plambeck et al. 1982; Masson et al. 1984).

\section{d) Similarities between Line Maps and Broad-Band Maps}

Since line emission accounts for much of the broad-band flux from Orion, then maps of emission in the dominant molecular species should look similar to the broad-band "continuum" maps. The line emission from $215-247 \mathrm{GHz}$ is dominated by $\mathrm{SO}_{2}(\sim 30 \%$ of the flux $)$ and $\mathrm{CO}(\sim 25 \%$ of the flux). $\mathrm{SO}_{2}$ emission is known to come from the plateau source which is spatially compact and located in the vicinity of IRc2. The CO emission has a large plateau component as well but also displays significant spatially extended emission. Other major molecular species (e.g., SO and $\mathrm{CH}_{3} \mathrm{OH}$ ) follow one or the other of these spatial distributions.

The $400 \mu \mathrm{m}$ map of Keene, Hildebrand, and Whitcomb (1982) displays this general pattern of spatially compact emission centered near IRc2 and additional extended emission. The chief difference is the presence in the $400 \mu \mathrm{m}$ map of a second compact source well to the south $\left(-5^{\circ} 25^{\prime} 50^{\prime \prime}\right)$. This southern source is prominent in some molecular line maps, particularly a ${ }^{13} \mathrm{CO}$ map of Schloerb and Loren (1982) and most strongly in an $\mathrm{HCO}^{+}(J=3-2)$ map of Wootten and Loren (1984).

\section{e) Other Molecular Clouds}

Similar results may hold for other molecular clouds. However, in cooler clouds with narrower line widths, the molecular 
ission would be restricted to the optically thick line cores.

Thus, for similar amounts of molecular material the line flux

, would be somewhat reduced relative to any true continuum 'on' flux.

II.

\section{CONCLUSIONS}

A high spectral density of emission lines is observed in Orion $\mathrm{A}$ at wavelengths around $1.3 \mathrm{~mm}$. These lines are sufficiently strong and broad that in many places they obscure the underlying continuum. The combined flux in the strongest of these lines is estimated to be approximately $40 \%$ of the broad-band flux. Accounting for weaker, presently undetected lines increases this to about $60 \%$. Within the errors, line emission could be the dominant contribution to the millimeter-wave flux.

Single-dish millimeter-wave astronomy at the Owens Valley Radio Observatory is supported by NSF grant AST-8214693. The authors would like to thank R. E. Miller of AT \& T Bell Laboratories, Murray Hill, for supplying the junctions used in this work. The authors are also grateful to D. P. Woody and S. L. Scott for their assistance and to Jocelyn Keene for helpful discussions.
Blake, G. A., et al. 1984, in preparation.

Elias, J. H., et al. 1978, Ap. J., 220, 25.

Friberg, P. 1984, Astr. Ap., 132, 265.

Keene, J., Hildebrand, R. H., and Whitcomb, S. E. 1982, Ap. J. (Letters), 252, L11.

Masson, C. R., et al. 1984, Ap. J. (Letters), 283, L37.

Plambeck, R. L., Wright, M. C. H., Welch, W. J., Bieging, J. H., Baud, B., Ho, P. T. P., and Vogel, S. N. 1982, Ap. J., 259, 617.

Schloerb, F. P., Friberg, P., Hjalmarson, A., Höglund, B., and Irvine, W. M. 1983, Ap. J., 264, 161

Geoffrey A. Blake and T. G. Phillips: Downs Laboratory of Physics 320-47, California Institute of Technology, Pasadena, CA 91125

C. R. MAsson: Downs Laboratory of Physics 405-47, California Institute of Technology, Pasadena, CA 91125

E. C. Sutron: Space Sciences Laboratory, University of California, Berkeley, CA 94720
Schloerb, F. P., and Loren, R. B. 1982, Symposium on the Orion Nebula to Honor Henry Draper, ed. A. E. Glassgold, P. J. Huggins, and E. L. Schucking, Ann. NY Acad. Sci., 395, 32.

Sutton, E. C., et al. 1984 , in preparation.

Westbrook, W. E., Werner, M. W., Elias, J. H., Gezari, D. Y., Hauser, M. G., Lo, K. Y., and Neugebauer, G. 1976, Ap. J., 209, 94.

Wootten, A., and Loren, R. B. 1984, in preparation. 